\begin{abstract}
Violence against women students is increasingly recognized as a significant public health and human rights issue. The Intervention Initiative is a facilitated bystander intervention educational program commissioned by Public Health England for use by all English universities to prevent violence, abuse and coercion. The success of the program with first-year Law students at a large university in the South West of England was evaluated through course evaluation feedback and in a questionnaire study. Student experience was exceptionally good across all measures. In paired sample t-tests, prosocial bystander behavior did not increase significantly from pretest to posttest immediately after taking part in the program. Rape myth acceptance, domestic abuse myth acceptance and denial decreased significantly $(\mathrm{p}<.001 ; d>.599)$. Bystander efficacy, readiness to help and responsibility increased significantly $(\mathrm{p}<.001, d=.408-.703)$ and intent to help increased significantly $(\mathrm{p}=.007, d=.248)$. Exposure to a concurrent social marketing campaign on campus had a significant strengthening effect on improvement of attitudes to rape myths $(p=.010)$ but not any other outcome measures. No significant backlash was identified.
\end{abstract}

Violence against women is a global public health problem (García-Moreno et al., 2013). There is a growing body of international evidence documenting universities as significant sites for violence against women (DeGue, 2014; Fisher, Cullen, \& Turner, 2000; Krebs, Lindquist, Warner, Fisher, \& Martin, 2007), where risk factors converge (Fenton, Mott, McCartan, \& Rumney, 2016; Powell, 2011; Schwartz, DeKeseredy, Tait, \& Alvi, 2001; Schwartz \& Pitts, 1995). Recently, an evidence base has begun to emerge from a number of surveys conducted in English universities indicating that violence against women is a serious problem with considerable impact for English university students in terms of their health and academic 
performance (NUS, 2011; Stenning, Mitra-Kahn, \& Gunby, 2012). Further, the cultural phenomenon of 'lad culture', perhaps akin to the American 'guyland' (Berkowitz, 2011) has been reported extensively in the popular media and in an associated body of sociological research, and is recognized as a concern for universities (e.g. Phipps \& Young, 2015).

\section{Bystander intervention}

A growing research base, predominantly from the US, indicates that bystander intervention shows particular aptitude for addressing prevention and response in university settings (DeGue, 2014; DeGue et al., 2014; Fenton et al., 2016). The rising importance of bystander programs is exemplified by US legislation which requires bystander programming in public and private colleges and universities participating in federal student aid programs (Campus SaVE Act, 2013). Bystander training aims to empower participants to become prosocial bystanders who recognize and intervene to prevent problematic behaviors which support violence or may become violent. By intervening, powerful messages are sent to wrongdoers, which, if constantly reinforced at community level, can shift social norms to exclude undesirable behavior.

Bystander models are complex and they address prevention at multiple levels. They aim to increase participants' likelihood of intervening and to deliver changes to attitudes, beliefs, social norms and peer group relationships with regard to gender roles and stereotyping, sexism, the acceptability of violence and rape myth acceptance that may (interrelatedly) affect conditions of perpetration and victimization. These aims of bystander programs relate to the outcome measures which have been commonly reported on in the literature. Indeed, a number of studies have reported statistically significant changes in: 1 . violence perpetration and victimization (Coker et al., 2016; Foubert, Newberry, \& Tatum, 2007; Gidycz, Orchowski, \& Berkowitz, 2011); 2. increased interventions made, and increased responsibility, confidence and intention to make 
interventions (Ahrens, Rich, \& Ullman, 2011; Amar, Sutherland, \& Kesler, 2012; Banyard, Moynihan, \& Crossman, 2009; Banyard, Moynihan, \& Plante, 2007; Banyard, Plante, \& Moynihan, 2005; Cares et al., 2015; Langhinrichsen-Rohling, Foubert, Brasfield, Hill, \& Shelley-Tremblay, 2011; Moynihan, Banyard, Arnold, Eckstein, \& Stapleton, 2011); 3. decreased rape myth acceptance and sexist attitudes, and decreased perception of peer sexist attitudes (Amar et al., 2012; Banyard et al., 2009; Banyard et al., 2007; Cares et al., 2015; Cissner, 2009; Coker et al., 2011; Foubert \& Newberry, 2006; Gidycz et al., 2011; Stewart, 2014); and 4. increased knowledge about violence, empathy towards survivors and decreased denial of violence as a problem (Amar et al., 2012; Banyard et al., 2005; Cares et al., 2015; Foubert \& Newberry, 2006; Gidycz et al., 2011). Some scholars have theorized and reported socalled 'backlash' effects (Flood, 2006; Hilton, 2000, Moynihan et al., 2011) from some short interventions, such as worsening attitudes (Winkel \& DeKleuver, 1997) or increased likelihood to engage in rape-supportive behaviors (Berg, Lonsway, \& Fitzgerald, 1999).

Recently, Coker and colleagues have reported on the distal outcome of a reduction of violence from a bystander program in a rigorous design over a 4-year period (Coker et al., 2016). A controlled trial across three comparable campuses found significant positive effects for victimization and perpetration at the community level suggesting efficacy of the bystander approach in changing culture on university campuses.

As most evidence emanates from the US, research is needed into the potential for effectiveness of bystander initiatives in other country university contexts (Fenton \& Mott, 2017).

\section{The Intervention Initiative (TII)}

TII (Fenton, Mott, McCartan, \& Rumney, 2014) was commissioned by Public Health England and is the first evidence-based bystander program for UK university settings. The 8- 
hour program is underpinned by a theory of change developed from evidence of the effectiveness of bystander programs as detailed above, integrated with theories of behavior change and of social psychological processes (Mott \& Fenton, 2014) and culturally adapted for a UK audience (Fenton \& Mott, 2017). The theory of change itemises the core immediate learning outcomes from the program (such as 'learning to identify violence - including warning signs, risk factors, trajectories and continuum') for individuals and then maps the different activities and processes that the program is designed for them to experience (such as 'desire to change own behavior'). The (measurable) intermediate outcomes for individuals include 'decreased rape and violence myth acceptance' and 'increased confidence to intervene'. The distal outcome is the 'decrease in community incidence of violence'.

During TII, participants are taken through the stages of bystander intervention moving from inaction to action (Latané \& Darley, 1969; 1970). Sessions 1-5 are concerned with knowledge, attitudes and beliefs, and aim to enable participants to recognize sexual and domestic violence as a problem and feel responsible for dealing with it. These sessions seek to generate an understanding of problematic behaviors along a continuum, risk factors, critical awareness of one's own gender inequitable attitudes, and empathy for victims. Sessions 5 to 8 correspond with the essential final stage of bystander intervention - that the bystander has the capacity and skills to be able to intervene safely and effectively (Banyard \& Moynihan, 2011; Powell, 2014). Experiential skills training allows participants to plan for and practice intervention skills for acting as an empowered bystander in their own social settings. The full program is available online at www.uwe.ac.uk/interventioninitiative and a detailed exposition of the program is available in Fenton and Mott (2017).

\section{The current study}


Bystander prevention research in the UK is far behind that of the US with no peer-reviewed published data from the UK available (Fenton et al., 2016). The main consideration for this first evaluation of a bystander approach in a UK university setting was to evaluate TII's effectiveness while allowing results to be comparable with other extant, similar or less similar prevention programs (see Schewe \& O'Donohue, 1993). The aim for this first evaluation of TII was to evaluate its effectiveness in this setting, measure any 'backlash' effects and assess potential interaction or signal-boosting effects of a concurrent student-led awareness raising campaign on campus. Effectiveness measures were based on expected outcomes in the theory of change model corresponding to existing scales reported upon in the literature. The initial intention for this study was to run a two group pre- post- design comparing intervention against control but we found that numbers of control group participants completing pretests and posttests were insufficient to justify detailed reporting. This study therefore uses a single group repeated-measures analysis. ${ }^{\mathrm{i}}$

A crucial component of this research was to address the sustainability of the program in the real world by measuring its acceptability to students. We theorized that for maximal effectiveness, a community-level intervention should be scheduled formally into university education rather than be delivered to self-selecting groups. Thus an important distinctive feature of this study was that $T I I$ was situated within the curriculum for the year cohort of Law students.

\section{Hypotheses}

We developed 10 hypotheses for this article. Our first hypothesis (1) was that students would evaluate TII positively for course content, efficacy and delivery, demonstrating acceptability to students of the TII program. The nine statistical hypotheses relate to the theory of change model (Mott \& Fenton, 2014). The scale and scope of this study defined the parameters of our statistical hypotheses, viz. a number of intermediate outcomes measured primarily in 
reported attitude change among students taking part in TII. Primary statistical hypotheses were that taking part in $T I I$ would show a positive benefit across a range of measures: (2) decreased rape myth acceptance; (3) decreased acceptance of myths concerning domestic abuse; (4) increased bystander efficacy; (5) readiness to help - reduced denial; (6) readiness to help increased responsibility; and (7) increased intent to help. We further hypothesized (8) that positive effects of the program would be enhanced among students who had been exposed to the campus awareness-raising campaign "The Anti-Abuse Campaign".

We had two secondary hypotheses. We hypothesized that: (9) students taking part in TII would report increased engagement in bystander intervention behavior. The secondary status of this hypothesis came from the knowledge that posttest timing would weaken the data. Our final hypothesis (10) was that 'backlash' (a significant negative change in attitude) would occur in no more than $10 \%$ of participants, or half the number of participants experiencing a significant positive change in attitude, whichever was the greater proportion. Hypothesis testing was bidirectional given the importance of capturing any (theoretically potential) negative effects.

\section{Method}

\section{Participants}

Participants were 354 first year students studying Law at a large university in the South West of England. The pretest was completed by 274 participants. 210 students completed the posttest, of whom 131 completed both questionnaires. 213 students completed the course evaluation feedback form. See Figure 1 for a diagram describing the flow of participants. The study was conducted in compliance with the University's Faculty of Business and Law Research Ethics Committee, endorsed and supported by Public Health England. Electronic informed consent was provided by each research participant prior to beginning the online questionnaire. 


\section{Procedure}

\section{Delivery of $T I I$.}

TII was timetabled within a core level one module for all Law students with academic credit available for reflective logs on each TII session as part of summative assessment. No other incentives were given. Students received a short motivational address encouraging them to attend and to participate in the evaluation. Students were advised about wellbeing issues and alternative assessments were in place.

TII was delivered in four 2-hour sessions to 14 groups of between 15 and 25 students between October 2014 and January 2015. A social marketing “Anti-Abuse” Campaign (https://www.thestudentsunion.co.uk/representation/campaigns/enditnow/) led by the Student Union took place on campus between November 2014 and January 2015.

\section{Student course evaluation.}

At the final session students were given a paper copy of the course evaluation feedback form. Anonymity was stressed. Completed forms were sealed in an envelope and given to the program administrator who collated the data.

\section{Questionnaire administration.}

All Law students were emailed a link to the questionnaire via the Qualtrics online survey platform. Data collection was closed after 17 days and before TII began. The posttest questionnaire included five additional questions and was administered in the same way one week after TII had been completed. Where possible, the order of the following scales, and items within scales, presented to participants was randomized to spread effects of survey fatigue.

\section{Measures}

\section{Course feedback evaluation form.}


Students used a five-point rating scale across all items, where $1=$ "definite no or not good" and $5=$ "definite yes or excellent" to rate the extent to which the program met its learning objectives (improved knowledge, attitudes, likelihood to intervene and leadership skills) and aspects of the program design and facilitation. Space was provided for comments.

\section{Main Questionnaire.}

Questions from the scales detailed below were modified where necessary for UK English language and cultural relevance. Full details are available from the first author.

\section{Rape and domestic abuse myth acceptance.}

We used McMahon's (2011) modified short form of the Illinois Rape Myth Acceptance (IRMA) scale to measure attitudes and beliefs about sexual violence. We also developed a fiveitem question set (unvalidated) to assess attitudes and knowledge about domestic abuse, using one relevant item from the Acceptance of General Dating Violence Scale (cited in Coker et al., 2011). Four additional items (such as “Domestic abuse doesn't really happen in young people's relationships") were developed by the authors based on the content of TII. On a scale of 1-5, higher scores represent higher myth acceptance. The mean IRMA score and mean Domestic Abuse Myth Acceptance score were calculated for each participant at pretest and posttest.

\section{Readiness to help.}

Following Moynihan et al. (2011) we adapted items forming two subscales - Denial and Responsibility - calculated originally from a factor analysis of the Readiness to Change Scale II. Items relating to domestic abuse were added to the items relating to sexual violence for both subscales (it should be noted that Banyard et al. (2014) have refined the Readiness to Help measures and their new scale relates to sexual violence/abuse, relationship abuse and stalking). Responses on the Denial subscale were coded for lower scores to indicate greater denial or lack 
of awareness. Responses on the Responsibility subscale were coded for higher scores to indicate a greater taking of responsibility. Mean scores for all items in the range 1-5 produced a Readiness to Help score.

Bystander efficacy.

Banyard, Moynihan and Plante's (2007) Bystander Efficacy Scale, with some modifications, was used to measure participants' self-reported confidence to perform bystander actions. One extra item was added, "Help a friend by providing information about specialist support services for domestic abuse" to correspond with program content. A mean score was calculated from each of the scores indicating confidence in the range 1-100.

\section{Intent to help.}

We used the Intent to Help Friends Scale and Intent to Help Strangers Scale (brief versions) (Banyard et al., 2014, with minor modifications) to calculate an overall Intent to Help score from the mean of each of the items in the range 1-5, with higher scores indicating greater intent to help.

\section{Bystander behavior.}

We used Banyard's Bystander Behavior Scale - Revised (BBS-R) (as modified by McMahon, Postmus, \& Koenick, 2011, with further minor modifications) to measure participants' engagement in a range of bystander behaviors in the previous two months. Five additional items borrowed from the work of Coker et al. (2011) were introduced and we followed the scoring protocol of McMahon et al. (2011) so that engaging in a behavior was scored +1 , not being in the relevant situation was scored 0 and being in the situation but not engaging in the bystander behavior was scored -1. A summative bystander behavior score was calculated for each participant at pretest and posttest. 


\section{Additional posttest questions including Anti-Abuse Campaign.}

Five questions were added to the questionnaire for the posttest administration in relation to attendance of TII. Zero attendance was an exclusion criterion. Students were asked whether they had been aware of / participated in the simultaneous awareness-raising program ("Anti-Abuse Campaign"). Responses were coded 1 for no awareness of the campaign and 2 for awareness, signing the campaign pledge or otherwise being involved in the campaign.

\section{Backlash.}

The method suggested by Moynihan et al. (2011) was used to measure potential attitude backlash. A categorical variable for each participant was computed for each of the four attitudinal variables: IRMA, domestic abuse myth acceptance, denial and responsibility. This variable represented the difference between pretest and posttest (pretest minus posttest score for IRMA and domestic abuse myth acceptance; posttest minus pretest for denial and responsibility). Any attitude change in a negative direction equal to or larger than one standard deviation from the study population was taken to indicate a substantial negative shift that could be attributable to backlash.

\section{Methods of Analysis}

Data validity checks, a missing values analysis, and descriptive summaries were performed prior to inferential analysis. Data screening did not show any unusual or unduly influential observations. The primary analysis for this pre-test post-test design used the paired samples $t$ test. The paired samples $t$-test is known to be robust to departures from normality in large sample sizes and retains good power advantages with large sample sizes displaying a positive correlation (e.g. Rasch \& Guiard, 2004). Effect size for the pre - post analysis was quantified using Cohen's $d$ (e.g. Rosenthal \& Rosnow, 1991); by way of general guidance, $d<0.1$ indicates a trivial 
effect, $0.1<d<0.3$ a small effect, $0.3<d<0.5$ a moderate effect, $0.5<d<0.8$ a medium effect, $0.8<d<1.3$ a large effect, and $d>1.3$ a very large effect and in practice $d>0.5$ is often looked upon as being the minimum threshold for a meaningful change.

Multiple imputations using chained equations [MICE] was used in a sensitivity analysis to determine the effect of non-response bias and these additional analyses did not alter substantive conclusions. On this basis the results provided are based on the participating sample without imputation. All analyses were performed in SPSS version 22.

\section{Results}

\section{Course feedback evaluation form}

There was a high response rate of $99 \%$ of those present at the final session, representing $74 \%$ of all students who were still enrolled in Law. There were no striking differences in the ratings of learning outcomes or program design and facilitation, by gender or by program facilitator. Results are reported in Fenton \& Mott (2015).

All learning outcomes received a mean score of more than four. All program design and facilitation items were rated more than four except "The length of the program and of each session was about right" which received a mean rating of 3.98. Written commentary indicates some students thought the sessions too short or too long. The average rating for recommending the program to others was 4.38 .

\section{Questionnaire and quantitative analysis}

\section{Outcome measures for the entire dataset.}

The missing value analysis conducted on all data did not show any significant differences between those who stayed in the study and those who did not complete all measures, and the data that were missing were found to be consistent with being missing at random. The data reported 
are from those participants who completed the study as described in Figure 1. The maximum amount of data available was used for each analysis.

Table 1 shows descriptive statistics for the outcome measures at pretest and posttest, for the students who completed both questionnaires. Given the range of individual responses, data for this group were subjected to further analysis using the paired-sample method in order to test the hypotheses. Missing data for paired-sample students on the variables analyzed for this article constituted $0.9 \%$. For comparison, Table 1 also shows the outcome measures at pretest and posttest for all students who satisfactorily completed the questionnaires. Missing data for all students on these variables constituted $4.5 \%$. Cronbach's alphas for all measures were very good $(.700-.928)$ with the exception of two datasets at pretest (Domestic Abuse Myth Acceptance: $\alpha$ $=.621$ among all students and Bystander Behavior: $\alpha=.605$ among paired samples) and one dataset at post-test (Denial: $\alpha=.693$ among paired samples).

\section{Paired sample tests for significant difference in outcomes.}

Table 2 shows descriptive statistics and statistical test results for significant difference in the outcome measures at pretest and posttest. Students who participated in the program showed significant improvement in the desired direction $(\mathrm{p}<.001)$ in the following outcome measures: rape myth acceptance; domestic abuse myth acceptance; bystander efficacy; readiness to help (both denial and responsibility). They showed significant improvement in the desired direction (p $=.007)$ in the measure for intent to help. The effect size measured by Cohen's $d$ was also above .5 for the measures of myth acceptance and readiness to help, while the effect size for efficacy was above .4, and above .2 for intent to help. There was no significant effect of the program on the students' reported engagement in bystander behavior $(p=.226)$.

\section{Effect of concurrent awareness raising campaign.}


Table 3 shows descriptive statistics and statistical test results for significant difference in pretest to posttest scores for students who were, or were not, exposed to the Anti-Abuse Campaign running on campus at the same time as the intervention. There was a significant result for the positive change in scores on rape myth acceptance $(p=.01)$, suggesting that exposure to the Anti-Abuse Campaign had a strengthening effect on the improvement of attitudes to rape myths resulting from participation in TII. Difference in the change on domestic abuse myth acceptance scores also approached significance. However, there were no significant effects found for the remaining outcome measures.

\section{Backlash.}

Figure 2 shows that at least $20 \%$ of participants showed scores that had improved by more than one standard deviation across all the attitude measures while between $2-4 \%$ showed scores that worsened by more than one standard deviation.

\section{Discussion}

The results of this study are promising and consistent with previous research. They indicate that $T I I$ has the potential for effectiveness. As the first study of bystander intervention in England, the findings represent the first empirical support from England to contribute to the published literature demonstrating the utility and potential effectiveness of bystander approaches in violence prevention in universities in local campus settings. The findings have additional importance because they demonstrate that significant results can be obtained from a cohort of students who did not self-select into a prevention program, and that $T I I$ is acceptable to students as a cohort in an English university setting.

\section{Course feedback evaluation form}


The course evaluation results were very positive, suggesting a high level of engagement with the aims of the program by students and recognition of the benefits for other students. The high response rate and anonymity give reason to trust the validity of the reported ratings. This result endorses the pedagogy underpinning the structural program design (Fenton \& Mott, 2017). This finding of acceptability to students is important for the sustainability of $T I I$ in the sector.

\section{Questionnaire and quantitative analysis}

\section{Primary hypotheses: paired sample tests for significant difference in outcomes.}

In line with our primary statistical hypotheses, the findings indicate that the program had a strongly significant effect on participants. The study findings correspond with the theoretical models upon which the program is predicated and are consistent with previous research. The program significantly decreased participants' rape and domestic abuse myth acceptance, and significantly increased their bystander efficacy and readiness and intent to help. All significant results also showed effect sizes indicating meaningful change with the highest effect sizes for myth acceptance and readiness to help, followed by efficacy and finally, intent to help.

This intervention is grounded in an understanding of a bystander program as a complex model. The first purpose of the program is to increase the ability of a bystander to identify and then intervene to prevent an act, situation or course of conduct occurring along the continuum of sexual violence. This approach appears successful as the findings demonstrate significant effects across a range of variables and factors which contribute to preparedness and increased likelihood of intervention (bystander efficacy; denial; responsibility; intent to help). The study adds to previous research which reports positive significant changes across steps towards intervening.

The second purpose of the program is its use as a strategic vehicle for the delivery of potential changes to attitudes and beliefs which enable violence against women and correlate 
with the stages of bystander intervention and conditions for perpetration of problematic behavior (e.g. Banyard \& Moynihan, 2011). The findings of significantly improved attitudes and beliefs as to rape and domestic abuse are consistent with previous research and support the utility of the program as based on a complex ecological model to address violence. There is little reason to support any alternative explanation for the significant changes between pretest and posttest, given that the results for the students in this study are consistent with findings from other studies. While demand characteristics are a possible explanation for some of the findings, it is reasonable to assume that demand characteristics (the desire to show researchers that the program is effective) would tend to have a similar effect upon responses to all questionnaire items across the board. However, responses were significantly different at posttest from pretest for attitudes while not significantly different for reported bystander behavior. Further research comparing responses of participants in different interventions, and where there have been no interventions, is needed.

\section{Effect of concurrent awareness raising campaign.}

The data suggest that the Anti-Abuse Campaign had a significant additive effect on positive change in students' attitudes towards rape myths. This effect is in line with an ecological theory of change that includes information about social norms as a factor contributing to attitudinal and or behavioral change (see e.g. Hester \& Lilley, 2014; Moosa, 2012). In other words, encountering the message that the community of students stand together against abusive behavior and reject rape myths helps to reinforce the 'top-down' learning occurring as a result of participation in TII. Other outcome measures were not significantly affected for this group although the general trend was in the expected direction.

\section{Secondary hypotheses: bystander behavior.}


The first interpretation from the null results is that $T I I$ was ineffective in producing behavior change. We cannot rule this out from the data. A second interpretation is that participants did not encounter opportunities to operationalize their learning at the point of posttest. In this study, posttest questionnaires were completed between one and eight weeks after the final two sessions of the program, in which intervention skills were learned (average completion of posttest $=$ last session + two weeks). Therefore the majority of participants only had two weeks in those two months during which they could have used any newly acquired intervention skills, as newly motivated prosocial bystanders. This interpretation could be tested by further evaluation of TII with follow-up of this cohort of students or post-testing other cohorts at later points.

A third interpretation is that the measure for bystander behavior (amended BBS-R) was not valid or reliable for our participants. We note that most of the original questions are framed in the language of 'acting in the moment' to challenge unacceptable behavior in a confrontational manner (e.g. "Confront a friend who is trying to have sex with someone who is practically unconscious"). TII by contrast encourages the use of body language, non-confrontational challenge such as distraction, interruption and changing the focus, and strategizing to intervene productively after the event. Additionally there are issues with the utility of using a simple additive method to score bystander behaviors and missed opportunities to intervene, especially in the context where opportunities (e.g. student parties; sexual encounters; friends in relationships) may be reasonably expected to be quite different between the 2-month period being recalled at pretest at the beginning of the first year, and at posttest in the middle of the first year.

More work is needed to adapt or develop a validated behavior measure better suited to use with TII. However, we also note two further difficulties with measuring bystander behavior. 
First, we suspect that situating one scale that purports to measure actual behavior within a long questionnaire populated largely by hypothetical attitudinal questions may confuse participants and solicit hypothetical answers. This suspicion was aroused on noting the high proportion of participants $(9.2 \%$, i.e. 32 students) who answered "Yes" when asked at pretest whether they had in the past 2 months reported a friend who committed a rape. This seems highly unlikely and it is more credible that this question was answered as though it was a hypothetical question despite its wording.

Second, the general ability of research participants to recall past behavior accurately is highly questionable (Bernard, Killworth, Kronenfeld, \& Sailer, 1984). We have found only one study which reports (relatively weak) significant change in measured bystander behavior (Banyard et al., 2007). Qualitative research may be more apposite for soliciting reliable information about behavior change (e.g. Banyard, et al., 2005).

\section{Backlash.}

In line with the secondary hypothesis, attitude backlash appeared minimal among the participants in $T I I$ while attitude improvement appeared substantial. Unlike most reported bystander program evaluations, participation in $T I I$ was not by self-selection. This fact enhances confidence that the extent of backlash that may be induced by TII is well within the bounds of acceptability. Backlash data compares well with reported backlash in the study by Moynihan et al. (2011), whose sample was self-selecting and entirely female.

\section{Limitations}

This study was conducted as an exploratory descriptive study using the cohort of participants (Law students at one university) who were the first to undertake the newly developed bystander intervention program. This cohort was not of a size to allow large scale 
analysis of clinical trial proportions and data from a matched control group of sufficient size were not available. Therefore while the results are indicative of very positive effects, the possibility that there may be the chance of false significant results within the data can not be discounted.

The statistical results from this study were consistent with results from similar studies of university bystander intervention programs, which may indicate generalizability in principle. Nonetheless, as this is the first study to be conducted upon students in an English university setting, the first to be conducted upon Law students in particular, and the first to be conducted upon students where the intervention was embedded within the curriculum, generalizability should be tested further.

Our sample size, which was further reduced by missingness between pretest and posttest data, precluded the generation of data subsets while other studies have shown that participant characteristics such as sex in particular, but also potentially ethnicity as well as year of study, can affect results (Banyard \& Moynihan, 2011; Brown, Banyard, \& Moynihan, 2014; Coker et al., 2014).

The student feedback data are original and therefore we cannot benchmark against previous research. Whilst the program was extremely well received by Law students at this university we cannot know whether these results are generalizable. The feedback data is limited to those still engaged with the course whose mean reported session attendance was $91.66 \%$ - and thus likely to be the most attentive, positive, enthusiastic students. However, as numbers in attendance at the final session of $T I I$ were not any lower (and anecdotally may well have been higher) than for comparable modules, it is possible that the absent students overlap with those who are poor attenders generally, rather than constituting a significant proportion of students with particular 
resistance or hostility to the program whose data may have skewed the results. This assumption should be tested in further research, as should baseline characteristics of poor attenders.

We also note that as the student feedback surveys - although anonymous - were not completed online but in the classroom in the presence of peers and the facilitator, the extent of demand characteristics may have been influenced.

\section{Methodological issues.}

The method we used to enable participants to self-generate 'unique' identifying codes proved unsatisfactory, generating a number of duplicate and irreconcilable response sets. We also note that the questionnaire was too long in its entirety, needing to be shorter in order to maximize completion and accuracy. Further consideration should be given to addressing the limitations of memory/recall questions for measuring behavioral outcomes such as bystander activity.

\section{Theoretical issues.}

For further evaluation of $T I I$, measures with improved construct validity of relevance to the theory of change for TII (Mott \& Fenton, 2014) should be developed. Such measures should capture specific pretest-posttest changes, for example in victim/survivor empathy, and should be further aligned with specific course content and learning outcomes.

\section{Conclusions and future directions}

Research into bystander programs for the prevention of violence against women in universities in the UK is in its infancy. This study is an important and timely step in the advancement of knowledge about the utility of an evidence-based bystander programming approach to prevention work in universities in England. This study provides an important model for ongoing research in localized university contexts in the UK. It is the first study to show 
significant improvements in an English university across a range of variables that are firmly associated in the literature with the occurrence of violence against women. Further, it shows the acceptability and feasibility of formal or curriculum based bystander programming in universities, in which the skills acquired are transferable and are skills for life. The success of TII provides evidence that bystander programming is an important part of a strategic response for universities (see Fenton \& Mott 2015b).

Further research is needed to test this program. Longitudinal multi-site testing using randomization, controls and incentivizing is indicated. With larger sample sizes it will be possible to shorten questionnaire length through a split-questionnaire design. When more UKbased research is conducted with TII, measures will require further development, using cognitive interviewing with UK students to enhance validity.

Broader issues identified with measuring outcomes in the field include the need to develop sound methodologies for capturing behavior change. Further theoretical and methodological development of the concept of backlash is required. It is accepted that some backlash is inevitable, indeed it can be a sign that culture change is occurring, but there appears to be little in the academic literature that formally theorizes or aims to measure backlash.

For bystander effectiveness to be meaningfully measured in future studies, new, rigorously tested standardized measures that capture information about all forms of violence against women need to be developed and adopted in the UK.

\section{References}

Ahrens, C. E., Rich, M. D., \& Ullman, J. B. (2011). Rehearsing for real life: The impact of the InterACT sexual assault prevention program on self-reported likelihood of engaging in 
bystander interventions. Violence Against Women, 17(6), 760-776.

doi:10.1177/1077801211410212

Amar, A. F., Sutherland, M., \& Kesler, E. (2012). Evaluation of a bystander education program. Issues in Mental Health Nursing, 33(12), 851-857. doi:10.3109/01612840.2012.709915

Banyard, V. L., Moynihan, M. M., \& Plante, E. G. (2007). Sexual violence prevention through bystander education: An experimental evaluation. Journal of Community Psychology, 35(4), 463-481. doi:10.1002/jcop.20159

Banyard, V. L., \& Moynihan, M. M. (2011). Variation in bystander behavior related to sexual and intimate partner violence prevention: Correlates in a sample of college students. Psychology of Violence, 1(4), 287-301. doi:10.1037/a0023544

Banyard, V. L., Moynihan, M. M., \& Crossman, M. T. (2009). Reducing sexual violence on campus: The role of student leaders as empowered bystanders. Journal of College Student Development, 50(4), 446-457. doi:10.1353/csd.0.0083

Banyard, V. L., Plante, E. G., \& Moynihan, M. M. (2005). Rape prevention through bystander education: Bringing a broader community perspective to sexual violence prevention. (No. 208701). Washington DC: US Department of Justice. Retrieved from https://www.ncjrs.gov/pdffiles1/nij/grants/208701.pdf;

Berg, D. R., Lonsway, K. A., \& Fitzgerald, L. F. (1999). Rape prevention education for men: The effectiveness of empathy-induction techniques. Journal of College Student Development, 40, 219-234.

Berkowitz, A. D. (2011). Using how college men feel about being men and "doing the right thing" to promote men's development. In J. A. Laker, \& T. Davis (Eds.), Masculinities in 
higher education: Theoretical and practical implications (pp. 161-176). New York:

Routledge.

Bernard, H. R., Killworth, P., Kronenfeld, D., \& Sailer, L. (1984). The problem of informant accuracy: The validity of retrospective data. Annual Review of Anthropology, 13, 495517.

Brown, A. L., Banyard, V. L., \& Moynihan, M. M. (2014). College students as helpful bystanders against sexual violence: Gender, race, and year in college moderate the impact of perceived peer norms. Psychology of Women Quarterly, 38(3), 350-362.

Campus SaVE act (2013): Violence against women reauthorization act of 2013, pub. L. 113-4, $\S 4,127$ stat. 54, (2013).

Cares, A. C., Banyard, V. L., Moynihan, M. M., Williams, L. M., Potter, S. J., \& Stapleton, J. G. (2015). Changing attitudes about being a bystander to violence: Translating an in-person sexual violence prevention program to a new campus. Violence Against Women, 21(2), 165-187. doi:10.1177/1077801214564681

Cissner, A. B. (2009). Evaluating the mentors in violence prevention program: Preventing gender violence on a college campus. New York: Center for Court Innovation. Retrieved from http://www.courtinnovation.org/sites/default/files/MVP evaluation.pdf

Coker, A. L., Cook-Craig, P. G., Williams, C. M., Fisher, B. S., Clear, E. R., Garcia, L. S., \& Hegge, L. M. (2011). Evaluation of green dot: An active bystander intervention to reduce sexual violence on college campuses. Violence Against Women, 17(6), 777-796. doi: $10.1177 / 1077801211410264$

Coker, A. L., Fisher, B. S., Bush, H. M., Swan, S. C., Williams, C. M., Clear, E. R., \& DeGue, S. (2014). Evaluation of the green dot bystander intervention to reduce interpersonal 
violence among college students across three campuses. Violence Against Women, 1-21. doi:1077801214545284

Coker, A. L., Bush, H. M., Fisher, B. S., Swan, S. C., Williams, C. M., Clear, E. R., \& DeGue, S. (2016). Multi-college bystander intervention evaluation for violence prevention. American journal of preventive medicine, 50(3), 295-302.

DeGue, S. (2014). Preventing sexual violence on college campuses: Lessons from research and practice. part one: Evidence-based strategies for the primary prevention of sexual violence perpetration. Washington DC: White House Task Force to Protect Students from Sexual Assault. Retrieved from https://notalone.gov/assets/evidence-based$\underline{\text { strategies-for-the-prevention-of-sv-perpetration.pdf }}$

DeGue, S., Valle, L. A., Holt, M. K., Massetti, G. M., Matjasko, J. L., \& Tharp, A. T. (2014). A systematic review of primary prevention strategies for sexual violence perpetration. Aggression and Violent Behavior, 19(4), 346-362. doi:10.1016/j.avb.2014.05.004

Fenton, R. A., \& Mott, H. L. (2015a). The intervention initiative: Student feedback February 2015. Bristol: University of the West of England. Retrieved from https://public.uwe.ac.uk/faculties/BBS/BUS/law/Law\%20docs/bystander/Partners/Studen t_Feedback_report.pdf

Fenton, R.A., and Mott, H.L. (2015b) Strategy for Addressing Sexual and Domestic Violence in Universities: Prevention and Response [online]. Bristol: University of the West of England. Available from:

www2.uwe.ac.uk/faculties/BBS/BUS/law/Law\%20docs/bystander/Partners/Strategy_for_ addressing_sexual_and_domestic_violence_on_universities.pdf 
Fenton, R. A., \& Mott, H. (2017). The bystander approach to violence prevention: Considerations for implementation in Europe. Psychology of Violence, 7(3), 450-458

Fenton, R. A., Mott, H. L., McCartan, K. \& Rumney, P. N. S. (2014). The intervention initiative toolkit and website. Retrieved from http://www1.uwe.ac.uk/bl/research/interventioninitiative/thetoolkit.aspx

Fenton, R. A., Mott, H. L., McCartan, K., \& Rumney, P. N. S. (2016). A review of evidence for bystander intervention to prevent sexual and domestic violence in universities. (No. 2016011). London, UK: Public Health England. Retrieved from https://www.gov.uk/government/uploads/system/uploads/attachment_data/file/515634/Ev idence review bystander intervention to prevent sexual and domestic violence in $\mathrm{u}$ niversities_11April2016.pdf

Fisher, B. S., Cullen, F. T., \& Turner, M. G. (2000). The sexual victimization of college women. research report. (No. NCJ 182369). Washington DC: ERIC. Retrieved from https://www.ncjrs.gov/pdffiles1/nij/182369.pdf

Flood, M. (2006). Changing men: Best practice in sexual violence education. Women Against Violence, 18, 26-36.

Foubert, J. D., Newberry, J. T., \& Tatum, J. (2007). Behavior differences seven months later: Effects of a rape prevention program. Journal of Student Affairs Research and Practice, 44(4), 1125-1146. doi:10.2202/1949-6605.1866

Foubert, J., \& Newberry, J. T. (2006). Effects of two versions of an empathy-based rape prevention program on fraternity men's survivor empathy, attitudes, and behavioral intent to commit rape or sexual assault. Journal of College Student Development, 47(2), 133148. doi:10.1353/csd.2006.0016 
García-Moreno, C., Pallitto, C., Devries, K., Stöckl, H., Watts, C., \& Abrahams, N. (2013). Global and regional estimates of violence against women: Prevalence and health effects of intimate partner violence and non-partner sexual violence. Geneva: World Health Organisation. Retrieved from http://apps.who.int/iris/handle/10665/85239

Gidycz, C. A., Orchowski, L. M., \& Berkowitz, A. D. (2011). Preventing sexual aggression among college men: An evaluation of a social norms and bystander intervention program. Violence Against Women, 17(6), 720-742. doi:10.1177/1077801211409727

Hester, M., \& Lilley, S-J. (2014). Preventing Violence Against Women: Article 12 of the Istanbul Convention: A collection of papers on the Council of Europe Convention on preventing and combating violence against women and domestic violence. Strasbourg: Council of Europe.

Hilton, N. Z. (2000). The role of attitudes and awareness in anti-violence education. Journal of Aggression, Maltreatment \& Trauma, 3(1), 221-238. doi:10.1300/J146v03n01_14

Krebs, C. P., Lindquist, C. H., Warner, T. D., Fisher, B. S., \& Martin, S. L. (2007). The campus sexual assault (CSA) study. Retrieved from https://www.ncjrs.gov/pdffiles 1/nij/grants/221153.pdf

Langhinrichsen-Rohling, J., Foubert, J. D., Brasfield, H. M., Hill, B., \& Shelley-Tremblay, S. (2011). The men's program: Does it impact college men's self-reported bystander efficacy and willingness to intervene? Violence Against Women, 17(6), 743-759. doi:10.1177/1077801211409728

Latané, B., \& Darley, J. M. (1969). Bystander" apathy". American Scientist, 57(2), 244-268. Latané, B., \& Darley, J. M. (1970). The unresponsive bystander: Why doesn't he help?. New York: Appleton-Century Crofts. 
McMahon, S. (2011). Rape myth beliefs and bystander attitudes among incoming college students. Journal of American College Health, 59 (1), 3-11. doi:10.1080/07448481.2010.483715

McMahon, S., Postmus, J. L., \& Koenick, R. A. (2011). Conceptualizing the engaging bystander approach to sexual violence prevention on college campuses. Journal of College Student Development, 52(1), 115-130. doi: 10.1353/csd.2011.0002

Moosa, Z. (2012). A theory of change for tackling violence against women and girls London: ActionAid/DFID/Gender and Development Network.

Mott, H. L., \& Fenton, R. A. (2014). Theory of change: The intervention initiative bystander intervention model for universities. Bristol: University of the West of England. Retrieved from http://eprints.uwe.ac.uk/28616/1/theoryOfChangeModel.pdf

Moynihan, M. M., Banyard, V. L., Arnold, J. S., Eckstein, R. P., \& Stapleton, J. G. (2011). Sisterhood may be powerful for reducing sexual and intimate partner violence: An evaluation of the bringing in the bystander in-person program with sorority members. Violence Against Women, 17(6), 703-719. doi:10.1177/1077801211409726

NUS (National Union of Students). (2011). Hidden marks: A study of women students' experiences of harassment, stalking, violence and sexual assault. London: NUS. Retrieved from http://www.nus.org.uk/Global/NUS_hidden_marks_report_2nd_edition_web.pdf;

Phipps, A., \& Young, I. (2015). Neoliberalisation and 'Lad cultures' in higher education. Sociology, 49(2), 305-322. doi: http://dx.doi.org/10.1177/0038038514542120 
Powell, A. (2011). Review of bystander approaches in support of preventing violence against women. (No. P-052-V B). Victoria, New South Wales: Victoria Health Promotion Foundation.

Powell, A. (2014). Bystander approaches: Responding to and preventing men's sexual violence against women. Melbourne, Australia: Australian Centre for the Study of Sexual Assault.

Rasch, D., \& Guiard, V. (2004). The robustness of parametric statistical methods. Psychology Science, 46, 175-208.

Rosenthal, R., \& Rosnow, R. L. (1991). Essentials of behavioral research: Methods and data analysis (2nd ed.). New York: McGraw Hill.

Schewe, P. A., \& O'Donohue, W. (1993). Sexual abuse prevention with high-risk males: The roles of victim empathy and rape myths. Violence and Victims, 8(4), 339-351.

Schwartz, M. D., \& Pitts, V. L. (1995). Exploring a feminist routine activities approach to explaining sexual assault. Justice Quarterly, 12(1), 9-31.

Schwartz, M. D., DeKeseredy, W. S., Tait, D., \& Alvi, S. (2001). Male peer support and a feminist routine activities theory: Understanding sexual assault on the college campus. Justice Quarterly, 18(3), 623-649.

Stenning, P., Mitra-Kahn, T., \& Gunby, C. (2012). Gender-based violence, stalking and fear of crime: Country report united kingdom. (EU-Project 2009-2011 No. JLS/ 2007/ISEC/415). Bochum; Keele: Ruhr-University Bochum; Keele University.

Stewart, A. L. (2014). The Men's project: A sexual assault prevention program targeting college men. Psychology of Men \& Masculinity, 15(4), 481-485. doi:10.1037/a0033947

Winkel, F. W., \& De Kleuver, E. (1997). Communication aimed at changing cognitions about sexual intimidation comparing the impact of a perpetrator-focused versus a victim- 
focused persuasive strategy. Journal of Interpersonal Violence, 12(4), 513-529.

doi:10.1177/088626097012004003

${ }^{\mathrm{i}}$ A more comprehensive report on the project and its findings including control group statistics is available from the authors: R.fenton@exeter.ac.uk and helen.mott.uk@gmail.com 